\title{
Indocyanine Green Fluorescence for Sentinel Lymph Node Detection in Early Breast Cancer
}

\author{
John Benson, MA, DM (Oxon), MD (Cantab), FRCS (Eng), FRCS (Ed) ${ }^{1,2}$ \\ ${ }^{1}$ Cambridge Breast Unit, Addenbrooke's Hospital, Cambridge University Hospitals NHS Foundation Trust, Cambridge, \\ UK; ${ }^{2}$ Anglia Ruskin University, Cambridge, UK
}

In this issue of Annals of Surgical Oncology, Sugie and colleagues report results of a prospective multicenter cohort study that further validates and assesses the accuracy of indocyanine green (ICG) for identification of sentinel lymph nodes (SLNs) in early breast cancer patients. ${ }^{1}$ SLN biopsy using the fluorescent tracer ICG combined with radioisotope avoids the need for blue dye and thereby minimizes potential morbidity, including cutaneous staining and allergic/anaphylactic reactions. Within the past 3-4 years there has been a significant increase in reports of allergic reactions to blue dye. The Medicines and Healthcare products Regulatory Agency (MHRA) issued a drug safety update in February $2012^{2}$ emphasizing that the occurrence of allergic reactions to blue dye was not uncommon and was estimated to have an incidence of $0.1 \%$ in the Axillary Lymphatic Mapping Against Nodal Axillary Clearance (ALMANAC) trial. ${ }^{3}$ In light of these recent concerns, there has been a trend away from the use of blue dye for SLN localization, and ICG as a tracer agent may serve as an adjunct to radioisotope in the first instance. This technology relies on generation of molecular fluorescence by contact of ICG with plasma proteins in the lymphovascular system. This fluorochrome absorbs light at a wavelength of approximately $800 \mathrm{~nm}$ with emission of a fluorescent signal when subatomic particles return from an excited to ground state. The illuminated subcutaneous lymphatic channels can be seen on a photodynamic eye (PDE) camera display and ICG tracked

(C) Society of Surgical Oncology 2015

First Received: 1 June 2015;

Published Online: 11 August 2015

J. Benson, MA, DM (Oxon), MD (Cantab), FRCS (Eng), FRCS (Ed)

e-mail: john.benson@addenbrookes.nhs.uk as it passes towards the axilla. The fluorescence is scattered by superficial tissues and cannot be detected at a depth of more than $1 \mathrm{~cm}$ with current equipment. The visual dimension of fluorescence with high optical sensitivity is a great advantage to radioisotope alone and could be complementary to radioisotope in the absence of blue dye.

A recently published systematic review found that ICG was more accurate than blue dye for SLN identification (odds ratio [OR] 18.37; $95 \%$ CI 8.83-39.10), with no statistically significant difference between ICG and radiocolloid for this metric (OR 0.81; $95 \%$ CI 0.03-24.29). ${ }^{4}$ This review highlighted the wide range of ICG doses used amongst studies, and standardization of dosage in terms of concentration and volume of ICG is essential to optimize performance of this technology. ${ }^{4}$ Nonetheless, there are potential drawbacks from the use of radioisotopes as a tracer agent for SLN localization, including cumulative radiation exposure for healthcare workers, issues with surgical waste disposal, and restrictions on access to radioisotopes secondary to mandatory licensing. Radioactive technetium-based nanocolloid is usually injected a few hours preoperatively in a dedicated nuclear medicine facility and requires a coordinated effort between different disciplines. Moreover, these radioisotopes are a by-product of a contracting nuclear industry, and supply might become unpredictable with more widespread usage, particularly within emerging economies of Asia. Therefore, potential problems exist with both standard tracer agents, and exploration of alternative, nonradioactive agents such as ICG and magnetic particles ${ }^{5}$ is warranted.

This is the largest study published to date assessing the accuracy of ICG for SLN identification. A total of 821 patients were available for final analysis from 12 centers throughout Japan, with all patients receiving a combination of fluorescent ICG and ${ }^{99} \mathrm{Tc}$ nanocolloid for localization. The authors chose as their primary endpoint the sensitivity 
of ICG for detection of positive nodes rather than rates of SLN identification which many other studies have adopted as their primary endpoint. However, both of these parameters are important but, ultimately, any new tracer agent must demonstrate at least comparable (noninferior) performance in terms of node positivity rates. An interim analysis was undertaken after the first 200 patients had been registered. The procedural detection rates for ICG and radioisotope were 96 and $97 \%$, respectively, with a node positivity rate of $25.5 \%$. It was concluded that detection rates for ICG and radioisotope were not significantly different and the study was rolled out to other centers, with eventual accrual of more than 800 patients.

Final results of this study have confirmed high rates of SLN identification (approaching $100 \%$ ) with ICG; $99.8 \%$ of patients had at least one fluorescent and/or radioactive node(s) and $97.2 \%$ of patients had at least one fluorescent node with no significant difference in detection rates for ICG and radioisotope ( $p=0.88$ ). Of note, the combination of ICG and radioisotope was significantly more sensitive than radioisotope alone $(p<0.001)$. Furthermore, for the primary endpoint of node positivity rates, there were no significant differences between these two tracer agents ( $p=0.18$ ) but a statistically significant improvement in detection of positive sentinel nodes with a combination of ICG and radioisotope compared with radioisotope alone (97.2 vs. $90.0 \% ; p<0.001$ ). These results echo those of Ballardini and colleagues from Milan ${ }^{6}$ showing a high level of concordance $(93.5 \%)$ between ICG and radioisotope for SLN detection. Some surgeons have expressed concerns regarding excessive numbers of nodes being fluorescent and therefore designated as sentinel, which would increase the average nodal yield and in turn the potential morbidity of the procedure. However, these concerns are not substantiated by recent publications reporting average nodal counts of less than 2 , which are comparable to those reported using conventional tracer agents. ${ }^{6-8}$ For example, in the ICG-10 trial $^{9}$ the average nodal count was 2.3, and the Milan group retrieved a total of 246 nodes amongst 134 patients (1.8 nodes per patient). ${ }^{6}$ In the current study, the number of fluorescent nodes removed (per patient) was significantly greater than the number of radioactivity nodes (2.3 vs. $1.7 ; p<0.001$ ), but this is not considered excessive and indeed may be advantageous in the post-Z0011 era when completion axillary dissection is selectively omitted. Fluorescence navigation provides a visual component that is lacking for radioisotope, and ICG permits a more orderly and sequential SLN dissection guided by visualization of lymphatic tissue. Identifying sentinel nodes in order of 'biological' priority is more difficult with radioisotopenodes are detected as hot spots irrespective of anatomical lymphatic flow. Nodes containing metastases are more likely to be the first node removed when ICG is the tracer agent. $^{1,9}$ A combination of radioisotope and ICG is preferable to the use of radioisotope alone, which can be surgically challenging and lead to more extensive dissection that is less focussed when there is no visual correlate to the tracer.

Although a nonrandomized study cannot demonstrate noninferiority, in the context of emerging data these results provide further evidence for the efficacy and safety of ICG as an alternative tracer agent for SLN biopsy. This combination of radioisotope and ICG could represent a transition phase, with ICG eventually becoming a sole tracer at a future stage when more clinical experience with its usage has accrued. It combines many of the advantages of blue dye and radioisotope without the disadvantages, particularly allergic reactions. This tracer combination would be more expensive than techniques involving blue dye, but provide an option for dual localization in the face of perceived problems with the latter. Use of radioisotope alone can be challenging for less experienced surgeons and in the longer term there is a need to explore novel tracer agents such as ICG. Evidence continues to accrue from other groups on the use of ICG for SLN localization, and an argument could now be made for performing SLN biopsy in routine breast cancer patients using a combination of radioisotope and ICG. Ideally, robust randomized trials should be conducted to assess ICG against current standard techniques, although inferiority margins are relatively small due to comparable performance characteristics. It is unlikely that identification rates and false negative rates would be impaired consequent to adoption of this technique with no evidence for any negative interaction between radioisotope and ICG.

It may eventually be possible to abandon radioisotope as a co-tracer and rely exclusively on ICG for sentinel node localization, which would dispense with any need for radioisotope facilities and mandatory licensing. It is hypothesized that fluorescence mapping can provide at least equivalent sentinel detection rates, but offers the opportunity for avoiding blue dye and could lead to improved cost effectiveness if radioisotope is eventually abandoned for routine SLN biopsy.

\section{REFERENCES}

1. Sugie T, Kinoshita T, Masuda N, et al. Evaluation of the clinical utility of the ICG fluorescence method in comparison to the radioisotope method for sentinel lymph node biopsy in breast cancer. Ann Surg Oncol (In press).

2. Medicines and Healthcare products Regulatory Agency. www. mhra.gov/Safetyinformation/DrugSafetyUpdate/. Accessed June 2015.

3. Mansel RE, Goyal A, Fallowfield L, et al. Sentinel node biopsy versus standard axillary treatment: results of the randomized multicentre UK ALMANAC trial. J Natl Cancer Inst. 2006;98: 599-609. 
4. Ahmed M, Purushotham A, Douek M. Novel techniques for sentinel lymph node biopsy in breast cancer: a systematic review. Lancet Oncol. 2014;15:351-62.

5. Douek M, Monneypenny I, Kothari A, et al. Sentinel node biopsy using a magnetic tracer versus standard technique: the SentiMAG multicentre trial. Ann Surg Oncol. 2014;21(4):1237-45.

6. Ballardini B, Santoro L, Sangalli C, et al. The indocyanine green method is equivalent to the Tc-labelled radiotracer method for identifying the sentinel lymph node in breast cancer: a concordance and validation study. Eur J Surg Oncol. 2013;39:1332-6.
7. Verbeek FP, Troyan SL, Mieog JS, et al. Near-infrared fluorescence sentinel lymph node mapping in breast: a multicentre experience. Breast Cancer Res Treat. 2014;143:333-42.

8. Schaafsma BE, Verbeek FP, Riebergen DD, et al. Clinical trial of combined radio- and fluorescence-guided sentinel lymph node biopsy in breast cancer. Br J Surg. 2013;100:1037-44.

9. Wishart GC, Jones LC, Loh S-W, Benson JR. Fluorescence mapping with indocyanine green (ICG) for sentinel lymph node detection in early breast cancer: results of the ICG-10 study. Eur J Surg Oncol. 2012;38:651-6. 